\title{
Design Of Thermoelectric Generator from Aluminum and Copper Elements
}

\author{
${ }^{1}$ M. A. Afolabi, and ${ }^{2}$ M. H. Ali ${ }^{*}$ \\ Department of Physics, Bayero University, Kano, Nigeria.
}

\begin{abstract}
The abundant heat accompanying the sunlight can be used to generate other forms of energy. Thermoelectric generator are important devices for this kind of means of harnessing the sun energy. A lot of heat are being wasted in industries that have machines that generate heat and in our homes during cooking. Thermoelectric generator is studied in this regard and a sample is designed and simulated for the purpose of utilizing this wasted energy. A temperature difference of up to $200^{\circ} \mathrm{C}$ is found to be generated from a $4 \mathrm{mV}$ electric potential by the designed TEG.
\end{abstract}

Keywords: Finite element method, Thermoelectric Generator (TEG), COMSOL Multiphysics, Renewable Energy

\section{Introduction}

The emerging global need for energy production, conservation, and management has intensified interest in more effective means of power generation. Enhancements to the existing energy supply must come from a variety of renewable sources including solar, wind, biomass, and others. Another potential source of power is electricity from heat sources through the use of thermoelectric materials. The heat can come from the combustion of fossil fuels, from sunlight, or as a byproduct of various processes (e.g. combustion, chemical reactions, and nuclear decay). Therefore, thermoelectric materials can play a role in both primary power generation and energy conservation (i.e. waste-heat harvesting).

The direct conversion of heat energy into electricity, or the reverse, by a semi- conductor thermoelectric power generation devices is related to electron transport phenomenon, and the inter related Seebeck, Peltier and Thomson effects [1]. Thermoelectric modules are devices that generate voltage and electrical power from temperature difference [2]. They are of great interest in energy application due to their well-known merit such as the absence of moving components, a reduction of maintenance, and an increase of system life. Its modularity allows for application in a wide scale range without significant loses in performance; the absence of working fluid avoids environmental dangerous leakage [3]. They are also direct (DC) sources and have capability of recovering the large amount of low grade waste heat [4]. They comprise $\mathrm{p}$ - and $\mathrm{n}$ - type semiconductor legs connected thermally in parallel and electrically in series, and sandwitched between two ceramic hot and cold plate [5]. Geometry optimization of the thermoelectric modules used as generator[6]. However, thermoelectric generators have also been used in a number of applications such as battery charging [7], waste heat recovery[8], power from radioisotopes [8], as well as electrification of rural homes [9].

In this work, COMSOL Multiphysics was used to study the thermoelectric capacity of Aluminum and Copper in constructing a dual purpose thermoelectric generator. The designed generator can be used in generating electric potential from temperature gradient as well as converting electric energy into thermal energy. The work involved preliminary experiment and simulation.

\section{Background Theory}

The thermoelectric couple field equations for the temperature $\mathrm{T}$ and electric potential $\mathrm{V}$ can be written for steady state calculation as

$$
-\nabla\left(\left(\sigma S^{2} T+\lambda\right) \vec{\nabla} T\right)-\vec{\nabla}(\sigma S T \vec{\nabla} V)=\sigma\left((\vec{\nabla} V)^{2}+S \vec{\nabla} T \vec{\nabla} V\right)
$$

$\vec{\nabla}(\sigma S \vec{\nabla} T)+\vec{\nabla}(\sigma \vec{\nabla} V)=0$

Where the material properties $S, \sigma$, and $\lambda$ enote Seebeck coefficiemt, the electric and thermal conductivity respectively. The above equation can be derived from the couple equation in [10].

*Corresponding author. Email address: alim@buk.edu.ng 


\section{The Finite Element Application (FEA) Model}

View FEA- program can handle thermoelectric effects, such as ANSYS [10] or COMSOL[11]. This work shows an implementation of thermoelectricity into COMSOL Multiphysics, which allows solving of common arbitrary partial differential equations (PDEs) of the field variable $U$ on a one to three dimensional regions [12]. Two PDE modes can be used. The "coefficient Form" and the "General Form". In the more didactical "Coefficient Form" PDE application mode, the program allows the definition of the coefficients for the following PDE:

$c_{a} \partial^{2} u / \partial t^{2}+d_{a} \partial u / \partial t+\nabla \cdot(-c \nabla u-\alpha u+\gamma)+\beta \cdot \nabla u+a u=f$

Equation (3) follows the notation of the COMSOL Multiphysics documentation which describes the PDE. The thermoelectric field equations can now be transformed into the "coefficient form" as follows. With the vector valued field variable $\mathrm{u}$, consisting of temperature $\mathrm{T}$ and $\mathrm{V}$,

$$
\vec{u}=\left(\begin{array}{l}
T \\
V
\end{array}\right)
$$

the coefficient $\mathrm{c}$ in (3) is

$$
c=\left(\begin{array}{cc}
\lambda+\sigma S^{2} T & \sigma S T \\
\sigma S & \sigma
\end{array}\right)
$$

and $\mathrm{f}$ is

$$
f=\left(\begin{array}{c}
\sigma(\vec{\nabla} V)^{2}+S \vec{\nabla} T \vec{\nabla} V \\
0
\end{array}\right)
$$

The other coefficients in equation (3) are zero for static calculations. These equatio form the basis for numerical computation of the potential as well as the temperature in COMSOL simulation results.

\section{Methodology}

A preliminary experimental work for thermocouple elements; copper and alumunum were carried out for single, double and triple junctions in series.

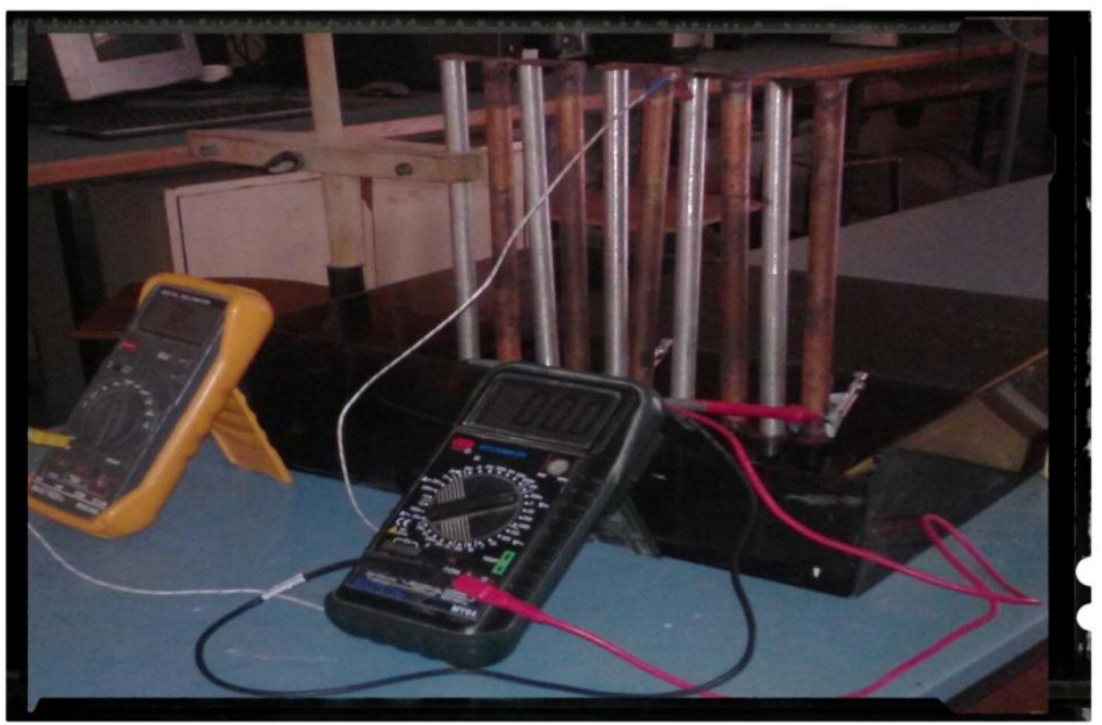

Plate 1. The set up for the Preliminary Experiment

Simulation results were also determined for thermocouple elements; copper and aluminum in COMSOL Multiphysics environment for single, double and triple junctions. The variation of temperature with the height of the thermocouple formed determined at contant potential difference in COMSOL Multiphysics environment. The effect of thickness on thermocouple was also determined with temperature at constant potential. The simulated results were used to design thermoelectric generator from twenty five (25) junctions 


\section{Result And Discussion}

A preliminary experimental and simulation work for thermocouple elements; aluminum and copper, were carried out for single junction in series by varying the output voltage against differential temperature which shows that as the output voltage increases, temperature gradient also increases.
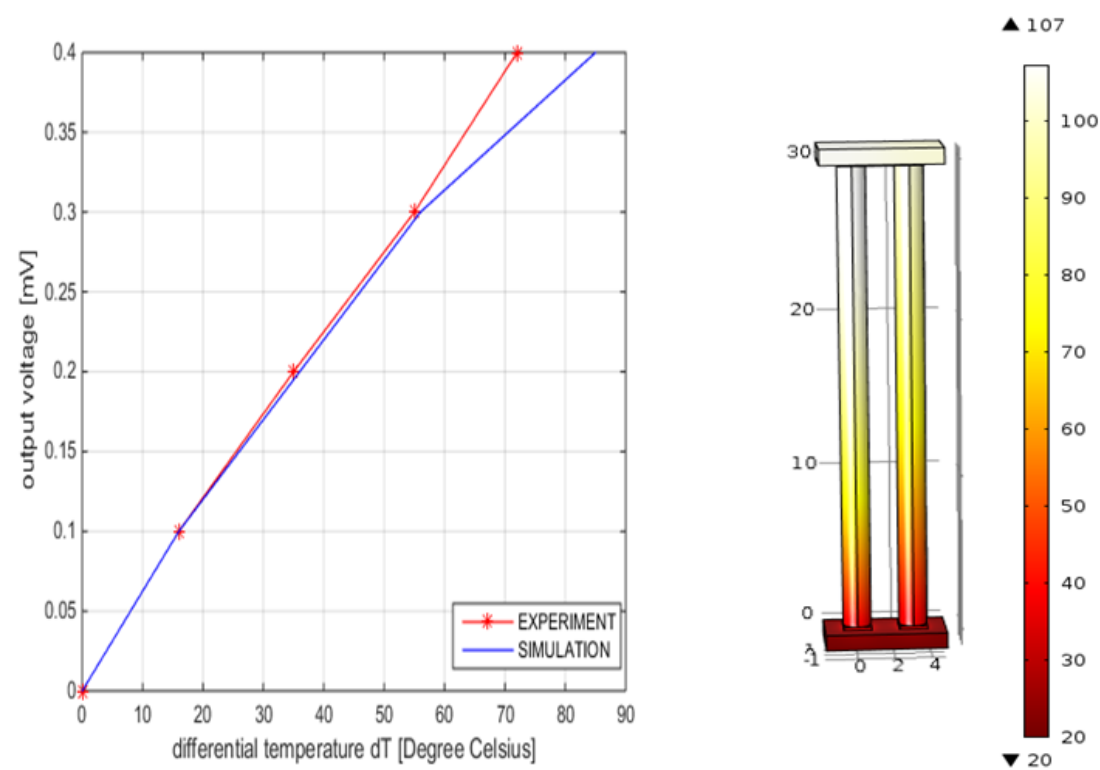

Fig. 1. A comparison of output voltage between the experiment and FEM simulation

A preliminary experimental and simulation work for thermocouple elements; aluminum and copper, were carried out for double junctions in series by varying the output voltage against differential temperature which shows that as the output voltage increases, temperature gradient also increases.
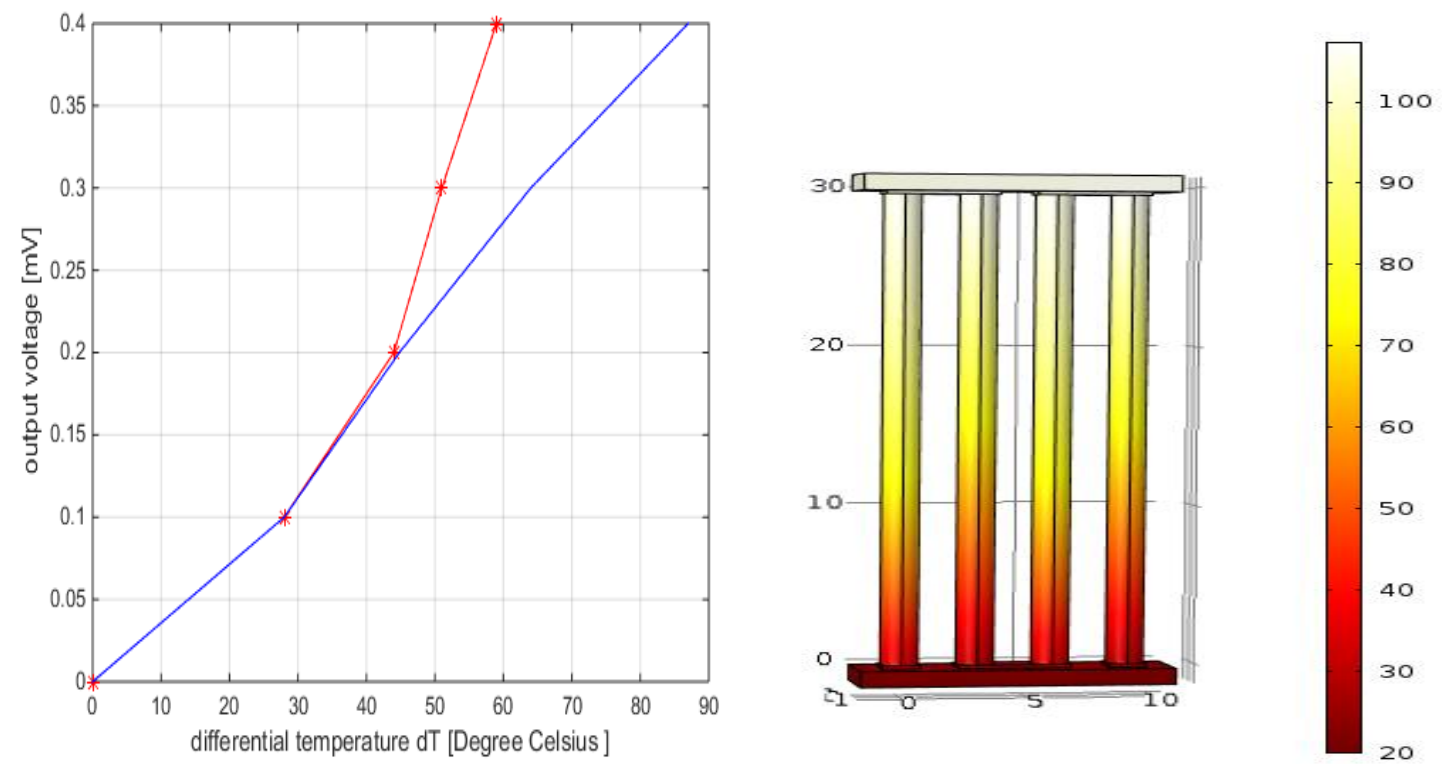

Fig. 2. A comparison of output voltage between the experiment and FEM simulation

A preliminary experimental and simulation work for thermocouple elements; aluminum and copper, were carried out for triple junctions junction in series by varying the output voltage against differential temperature which shows that as the output voltage increases, temperature gradient also increases. 

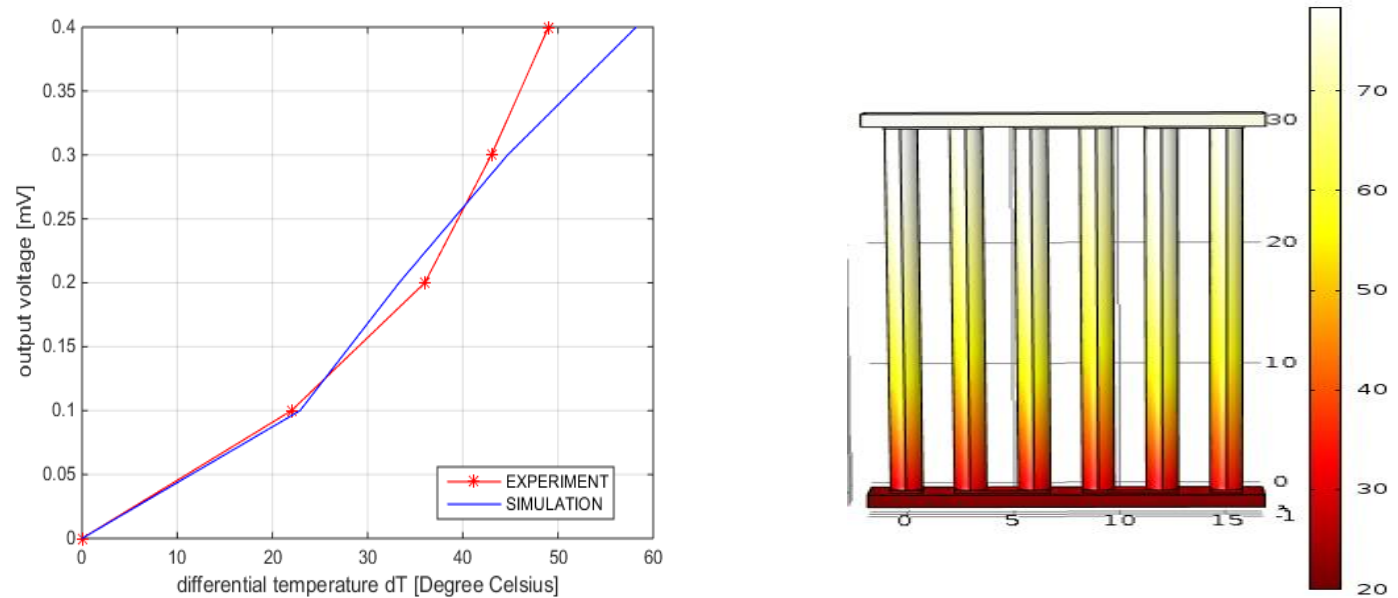

Fig. 3. A comparison of output voltage between the experiment and FEM simulation

The variation of temperature with the height of thermoelectric elements; aluminum and copper in series were measured for single junction which shows that as the height increases, temperature decreases at constant potential difference in COMSOL Multi-physics environment.
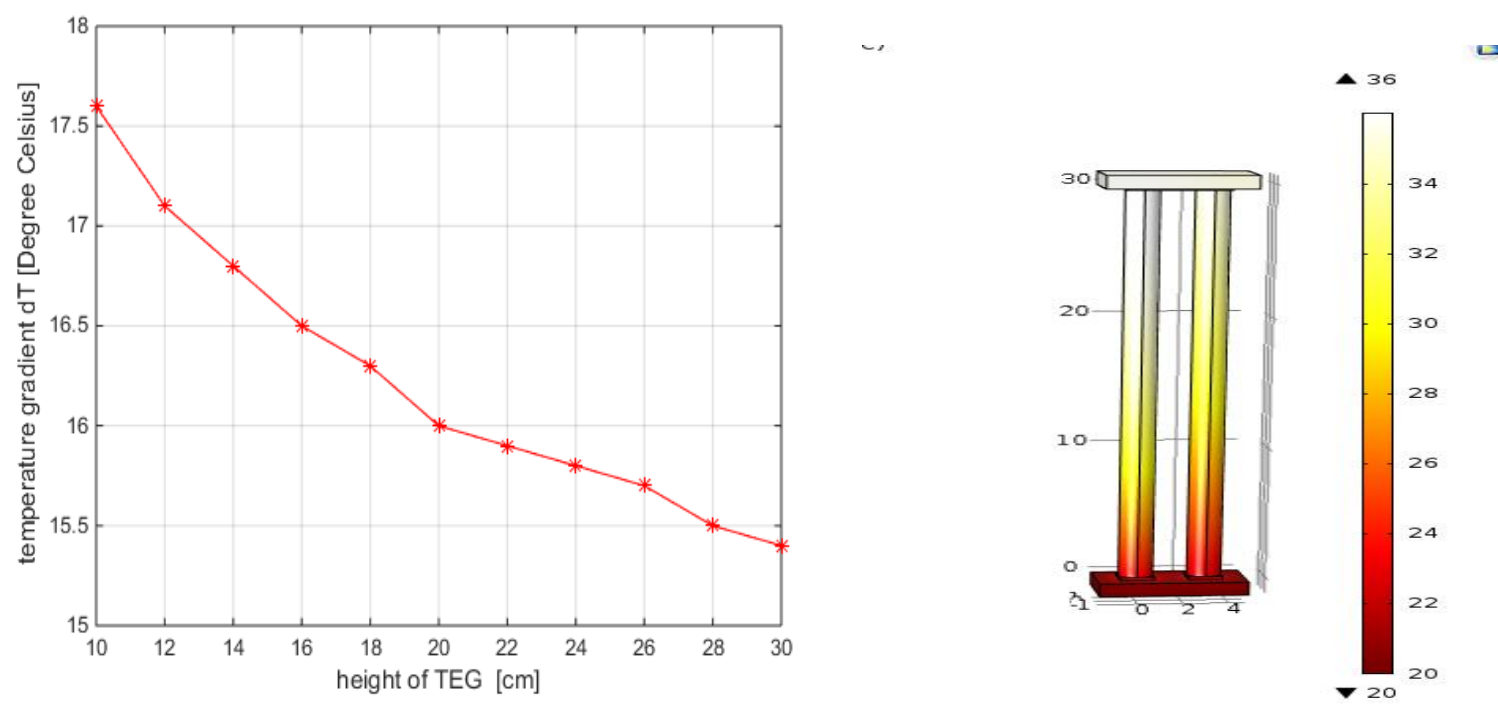

Fig. 4. A graph of differential temperature vs. height of TEG

The variation of temperature with the height of thermocouple elements; aluminum and copper in series were measured for single junction which shows that as the height increases, temperature decreases at constant potential difference in COMSOL Multi-physics environment.
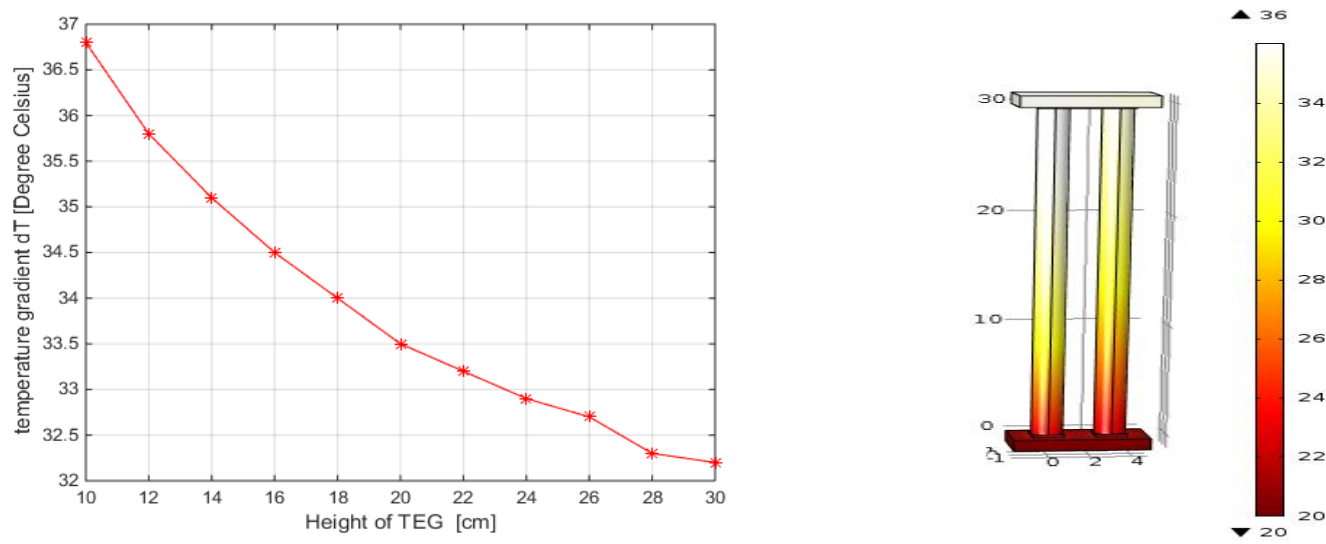

Fig. 5. A graph of differential temperature vs. height of TEG 
The variation of temperature with the radius of thermocouple elements; aluminum and copper in series were measured for single junction which shows that temperature increases with radius of the thermocouple at constant potential difference in COMSOL Multi-physics environment.
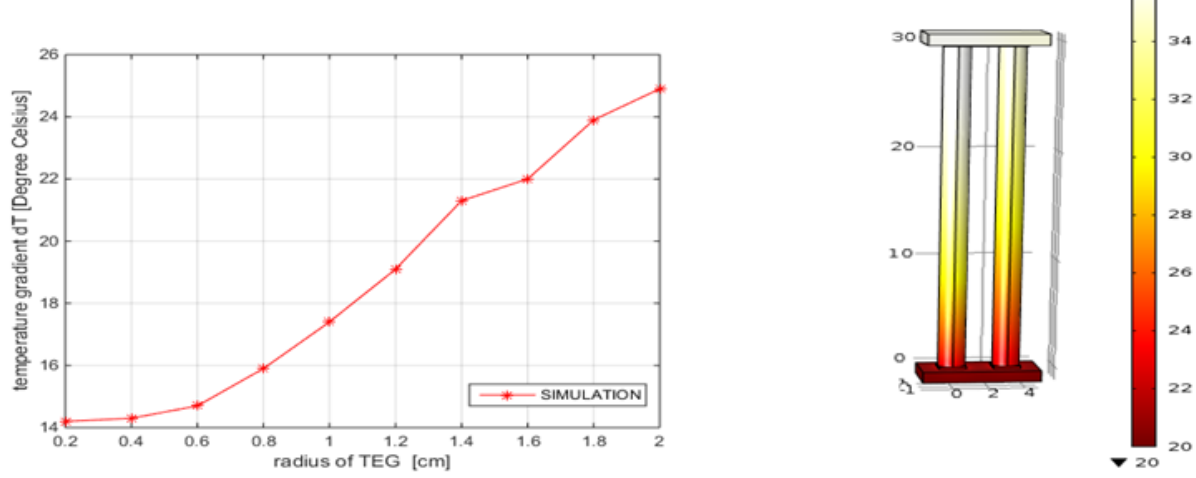

Fig. 6. A graph of differential temperature vs. radius of TEG

The variation of temperature with the radius of thermocouple elements; aluminum and copper in series were measured for single junction which shows that temperature increases with radius of the thermocouple at constant potential difference in COMSOL Multi-physics environment.
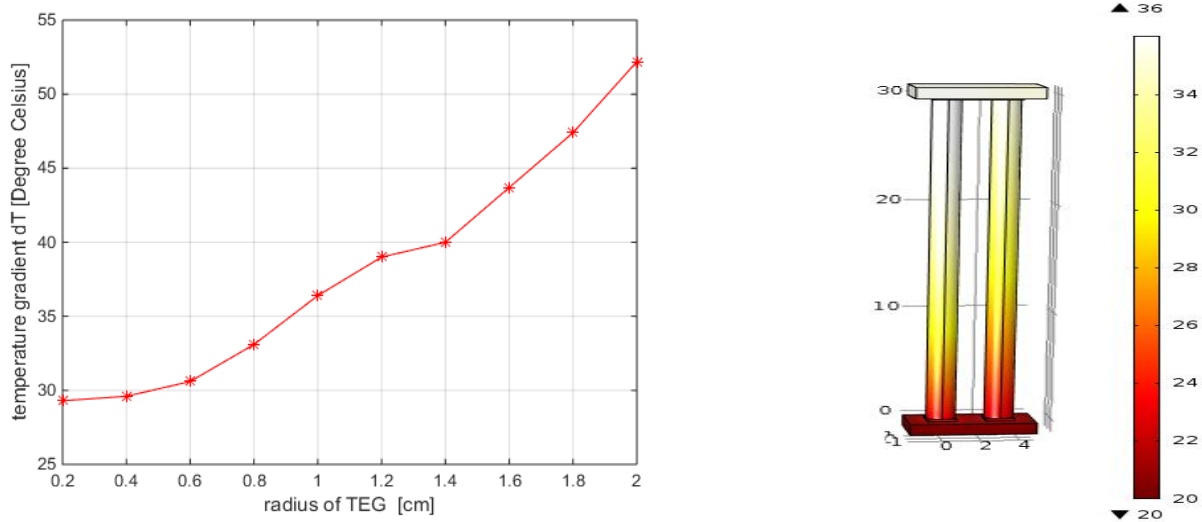

Fig. 7. A graph of differential temperature vs. radius of TEG

The variation of temperature with output voltage of thermocouple elements; aluminum and copper were measured for twenty five (25) junctions which shows that temperature increases with output voltage of the thermocouple using COMSOL Multi-physics environment.
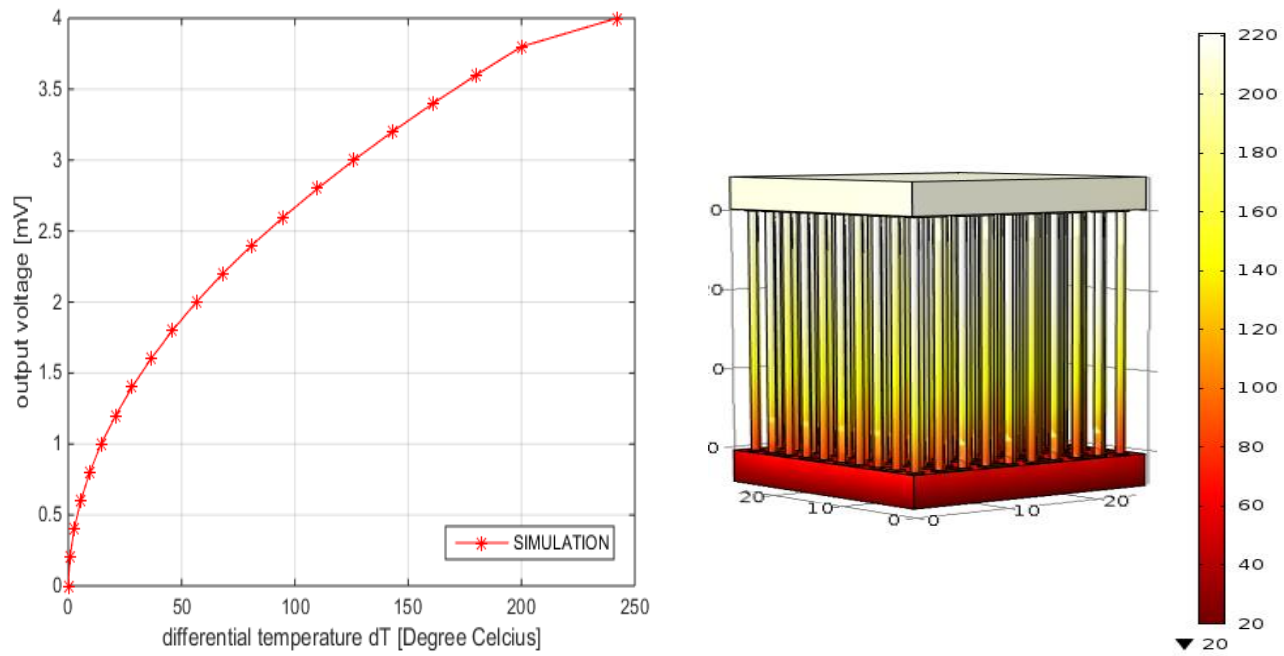

Fig. 8. A graph of differential temperature vs. output voltage 


\section{Conclusion}

In this work, we found that an electric potential of about $4 \mathrm{mV}$ can be able to generate temperature gradient of $200^{\circ} \mathrm{C}$ using the designed thermoelectric generator. This kind of device can be used to generate electric current from the heat of industrial engines and our stoves at homes. The generated electric energy can be used in charging batteries as well as electrification of rural homes.

\section{References}

[1]. D. M. Rowe, Handbook of Thermoelectrics. CRC press, 1995

[2]. M. Chen, S. Member, L. A. Rosendahl, T. J. Condra, J. K. Pedersen, and S. Member, "Numerical Modeling of Thermoelectric Generators With Varing Material Properties in a Circuit Simulator," vol. 24, no. 1, pp. 112-124, 2009.

[3]. M. Chen, L. A. Rosendahl, and T. Condra, "International Journal of Heat and Mass Transfer A three-dimensional numerical model of thermoelectric generators in fluid power systems," Int. J. Heat Mass Transf., vol. 54, no. 1-3, pp. 345-355, 2011.

[4]. X. Gou, H. Xiao, and S. Yang, "Modeling, experimental study and optimization on low-temperature waste heat thermoelectric generator system," Appl. Energy, vol. 87, no. 10, pp. 3131-3136, 2010.

[5]. S. B. Riffat and X. Ma, "Improving the coefficient of performance of thermoelectric cooling systems : a review," vol. 768, no. September 2003, pp. 753-768, 2004

[6]. S. B. Riffat and X. Ma, "Optimum selection ( design ) of thermoelectric modules for large capacity heat pump applications," no. December 2003, pp. 1231-1242, 2004.

[7]. J. Eakburanawat, "APPLIED Development of a thermoelectric battery-charger with microcontroller-based maximum power point tracking technique," vol. 83, pp. 687-704, 2006.

[8]. R. G. Lange and W. P. Carroll, "Review of recent advances of radioisotope power systems," vol. 49, pp. 393-401, 2008.

[9]. E. Taglialavore, S. Gortari, and M. G. Molina, "Development of thermoelectric generators for electrification of isolated rural homes," Int. J. Hydrogen Energy, vol. 35, no. 11, pp. 5818-5822, 2010

[10]. E. E. Antonova, D. C. Looman, and T. Drive, "Finite Elements for Thermoelectric Device Analysis in ANSYS," 2005

[11]. M. Jaegle, "Multiphysics simulation of thermoelectric systems," Eur. Conf. Thermoelectr. ..., no. 6, pp. 1-6, 2008.

[12]. R. L. F. P. P. Silvester, "Finite elements for electrical engineers." University Press, Cambridge, 3rd Edition, 1996. 